\title{
EU-IndiaGrid2 sustainable e-Infrastructures across Europe and India
}

\author{
Alberto Masoni \\ INFN Sezione di Cagliari \\ Cittadella Universitaria di Monserrato, 09042 Monserrato, Italy \\ Alberto.masoni@ca.infn.it
}

EU-IndiaGrid2 - Sustainable e-infrastructures across Europe and India - is a project funded by European Commission under the Research Infrastructure Programme of the Information and Society Directorate General with the specific aim of promoting international interoperation between European and Indian e-Infrastructures. 2010 has been an eventful year for eInfrastructures across Europe and India with a number of important achievements. EUIndiagrid2, basing on the achievements of the previous EU-IndiaGrid project and on the active role of its partners was at the core of all these events that contributed significantly to the project progress towards its objectives. The present article reviews the most recent e-Infrastructures developments in India and their relationship with respect to Europe and the Asia-Pacific area.

The International Symposium on Grids and Clouds and the Open Grid Forum Academia Sinica, Taipei, Taiwan

March 19 - 25, 2011 


\section{Introduction}

EU-IndiaGrid2 - Sustainable e-infrastructures across Europe and India - is a project funded under the Seventh Framework Programme of the European Commission started on January 2010. The project capitalises on the success of the previous EU-IndiaGrid project and has four main objectives:

O1)Consolidate \& enhance cooperation between European and Indian eInfrastructures for the benefit of EU-Indian collaboration in e-Science

O2) Support specific user communities in the exploitation of grid infrastructure in areas strategic for EU-Indian collaboration

O3)ensure a sustainable approach to e-Infrastructures across Europe and India through dissemination actions, meetings \& workshops

O4)foster and enhance cooperation with other European Initiatives in the Asian region and worldwide

Objectives O2,O3,O4 are functional to Objective O1) which represents the project main target. In the context of its activity the project strongly interacted with the main actors of the eInfrastructures scenario in India and Europe as well as in the Asia-Pacific area. In the following sections we will describe the e-Infrastructures scenario in India, its relationship with the situation in Europe and in Asia-Pacific, the specific role and activities of the EU-IndiaGrid2 project.

\section{2. e-Infrastructures in India}

The National Knowledge Network (NKN) [1] represents the main innovation in the Indian e-Infrastructure scenario. NKN is a high bandwidth and low latency network, with three layers (see figure 1). A powerful CORE with 7 Supercore locations with fully meshed multi-10Gbps connectivity and 26 Core locations having multi-10Gbps partially meshed connectivity with Supercore locations. A Distribution layer connected to the network core using multiple links at 2.5/10 Gbps. The Edges networks that connect institutions at 1 Gbps.

The creation of NKN was recommended by the Office of the Principal Scientific Adviser to Government of India and the National Knowledge Commission and obtained full approval with a budget of over 1 billion euro and a duration of 10 years by Government of India in March 2010. The National Informatics Centre (NIC) a premier Government Institution, is in charge of the implementation. NKN project is aimed at establishing a strong and robust internal Indian network capable of providing secure and reliable connectivity. NKN brings together all the main stakeholders from Higher Education, Science, Technology, Healthcare, Agriculture, Grid Computing, e-Governance. The project aims also at facilitating connection between different sectoral networks in the field of research, education, health, commerce and governance. 


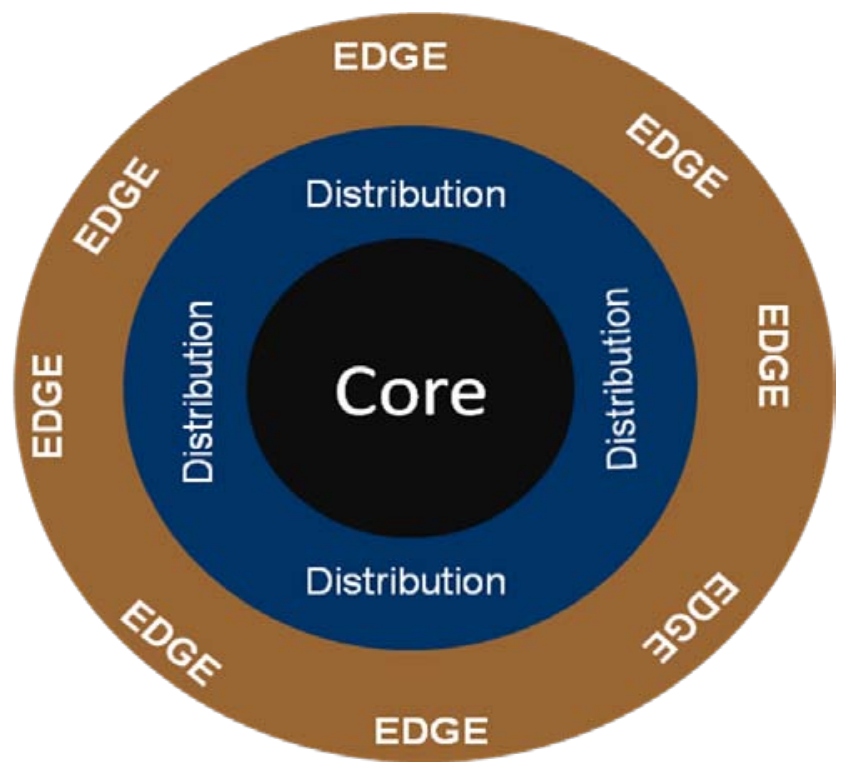

Figure 1: NKN Layers

This approach responds to a vision where different activities in the research domain but also other areas as Healthcare, or e-Governance can move from a silos-like structure to a gradual share of the upper layers from the network to the computing (grid) and the data management. This e-Infrastructure can provide a core of services not affordable to an individual application jumping across geographical, administrative and academic boundaries (see figure2).

The NKN infrastructure is entirely fiber based and owned by Government of India. It relies on a high capacity highly scalable backbone and covers the entire country. NKN will connect more than 5000 sites across the country serving million of end-users and all major escience projects. In the vision of Prof. Raghavan, Scientific Secretary to Principal Scientific Adviser to Government of India and Chief Architect and Chairman of Technical Advisory Committee of NKN, NKN represents for education a great integrator, making reachable and available the collective wisdom of institutions and laboratories with every Indian, irrespective of the geographical location, being able to benefit by accessing this vast intellectual resource. These features are also of paramount impact for research and for health related applications. In this case NKN provides is "Clear Visibility" of whatever medical records are generated at the remote end X Rays, 2D and 3D,MRIs, CT Scans, PETs and so on. Moreover thanks to the very high bandwidth and very low latency a patient requiring critical attention and an expert opinion can be remotely seen, examined, diagnosed, and treated. 

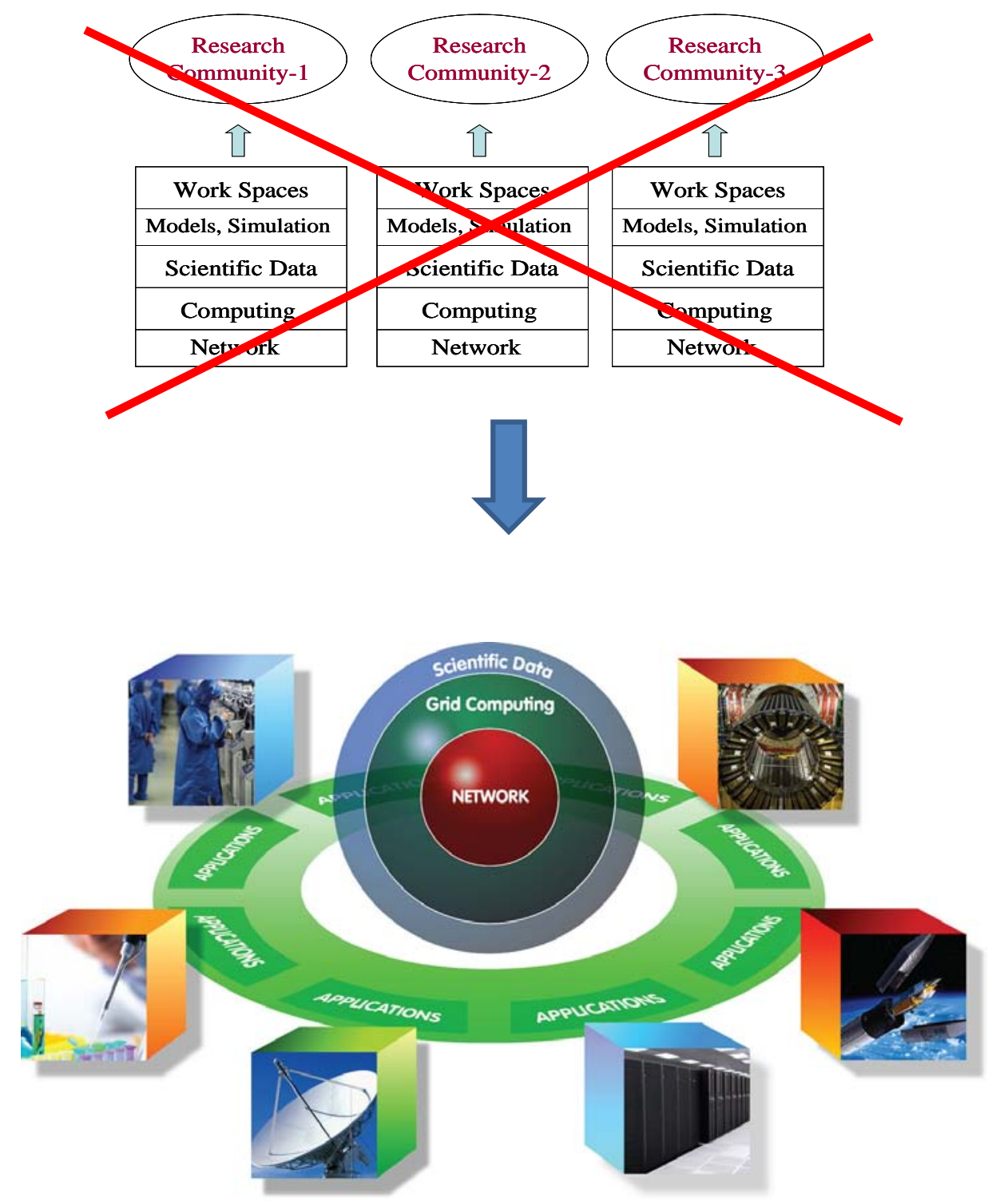

Figure 2, From a silos-like to integrated core of shared advanced services.

NKN implementation strategy consists of two phases the initial phase and the final phase. Initial phase is already operational with a fully meshed core backbone spanning across the country with twenty-three points of presence (PoPs) and connecting 90 institutions. Efforts are underway to scale the number of institutions connected to 550 in the next future.

In the initial phase NKN is already providing services for virtual classrooms and grid computing applications such as High Energy Physics to Climate modelling and Health care as well as collaborative design of advanced complex engineering systems. 


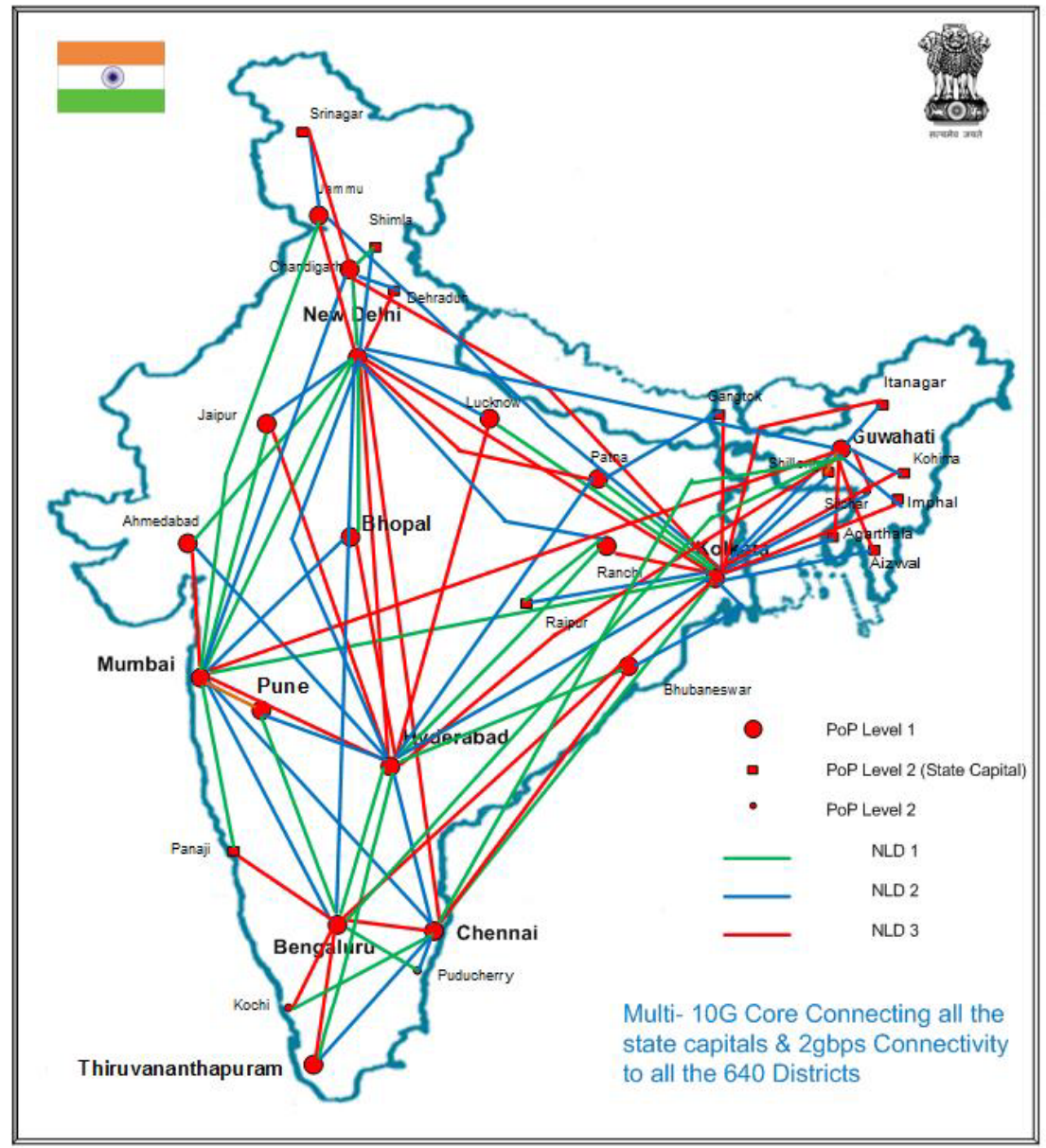

Figure 3 : NKN Phase 1

\section{International Connectivity}

NKN is connected to the Pan-European Research network, GÉANT [2], by means of a 2-5 Gbps link co-funded by Government of India and the European Commission within the framework of the TransEurasia Information Network project phase 3 (TEIN3)[3]. TEIN3 provides a link from Europe to Mumbai and also a 2.5 Gbps link from Mumbai to Singapore. In this vision, for research collaborations, Mumbai represents, at the same time, the Gate of India and the gateway to Asia-Pacific area.

In addition it is under way an agreement to connect the Open Science Grid in the USA in cooperation with the USA National Science Foundation and to connect to Japan a dedicated high speed link from Chennai. The NKN-TEIN3 connectivity is successfully exploited by several research applications as described in the sections below. 


\section{Grid Infrastructures}

Two main Grid Initiatives are present in India: the Regional Component of the WorldWide LHC Computing Grid (WLCG) [4] and the GARUDA National Grid Initiative [5]. Both are strongly connected with the EU-IndiaGrid2 project thanks to the presence, as project partners of the leading actors of both initiatives.

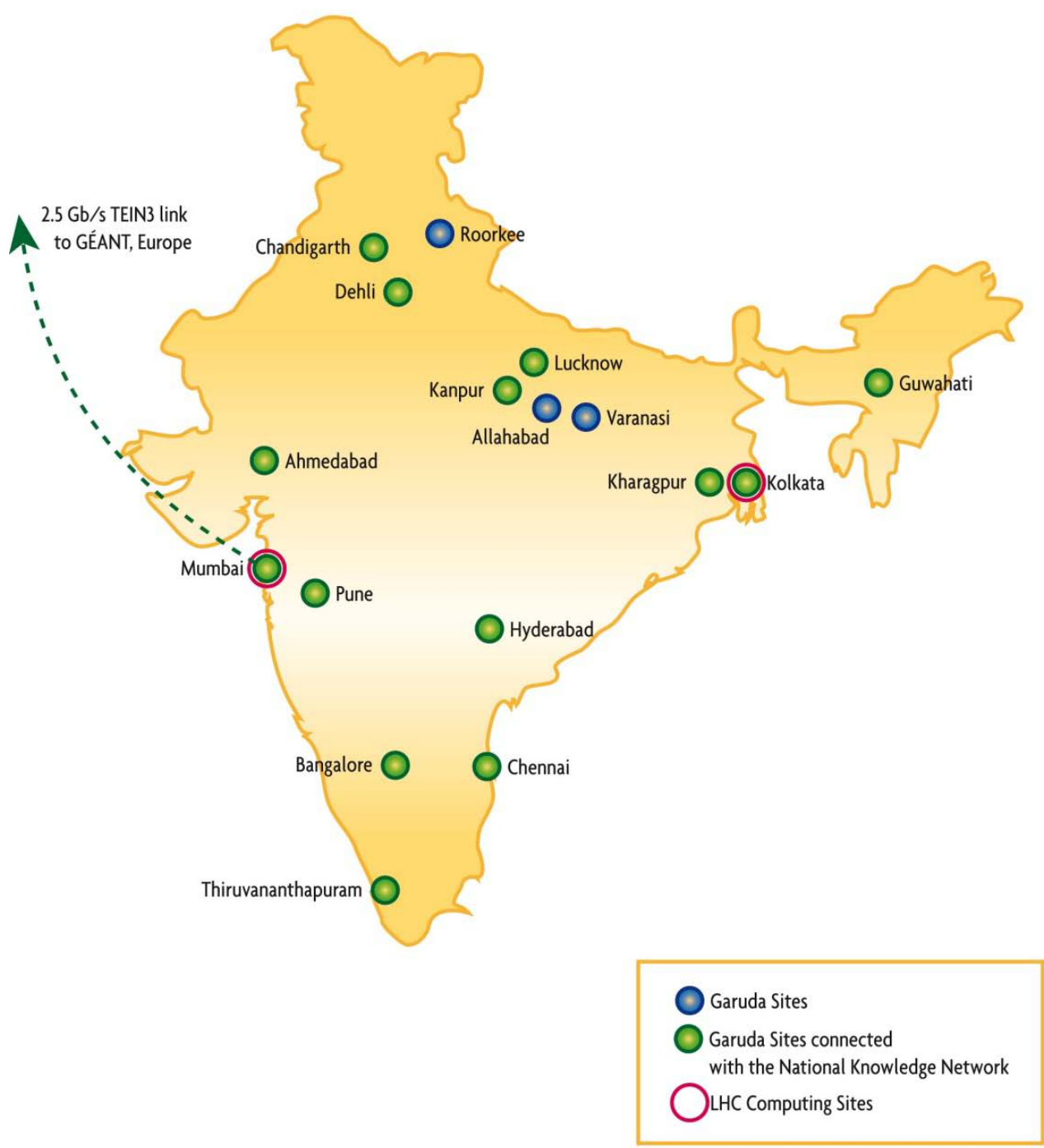

Figure 4: GARUDA Grid and WLCG Tier2 sites

\subsection{The Worldwide LHC Computing Grid in India}

WLCG represents the world largest grid infrastructure and with the start of data taking at the CERN Large Hadron Collider (LHC) WLCG entered the full production phase [6]. The data from the LHC experiments will be distributed around the globe, according to a four-tiered model. India participates with two Tier2 centres supported by the Department of Atomic Energy 
(DAE). One at the Tata Institute for Fundamental Research (TIFR) Mumbai provides the services for the CMS experiment. The other one, dedicated to the ALICE experiment, is located in Kolkata and managed by the Saha Institute of Nuclear Physics in collaboration with the Variable Energy Cyclotron Centre (VECC).

These Tier2 centres provide access to CMS \& ALICE users working from Tier III centres of Universities and national labs and LCG Data Grid services for analysis. TIFR is presently connected to CERN with the $2.5 \mathrm{~Gb} / \mathrm{s}$ TEIN3 link via NKN. The ALICE TIER2 centre is also connected via NKN at $1 \mathrm{~Gb} / \mathrm{s}$. Thanks to a coordinated actions of the main actors involved participating also to the EU-IndiaGrid2 project TIFR is successfully exploiting the TEIN3 connectivity for LHC data transfer since December 2010.

\subsection{GARUDA: the National Grid Initiative of India}

GARUDA is India's first national grid initiative bringing together academic, scientific and research communities for developing their data and compute intensive applications with guaranteed Quality of Service. The project is coordinated by Centre for Development of Advanced Computing (CDAC), which established also, in 2008, the Indian Grid Certification Authority (IGCA) for the first time in India. GARUDA ended its Proof of Concept phase in 2008 and concluded the Foundation Phase in August 2009. The project is currently in its third (Operation) phase which is focused on grid enabling specific applications of national priority as Disaster Management (DMSAR) and Bio informatics. At present GARUDA network infrastructure is provided by NKN, consolidating this way the integration between NKN and Indian grid infrastructures and related applications.

\section{The Role of EU-IndiaGrid2 Project}

EU-IndiaGrid2 is strongly integrated in the Indian e-Infrastructure scenario. Its partners take leading roles in NKN, WLCG and GARUDA and a solid and fruitful cooperation has been established between these initiative and the EU-IndiaGrid2 project. During its first 15 months of activity EU-IndiaGrid2 provided specific support to ensure exploiting the progress in connectivity favoring Euro-India cooperation in e-Science. The project supports the interoperation and interoperability between the European and the Indian grid infrastructures as well as four main application areas in the domain of Biology, Climate Change, High Energy Physics and Material Science. The main landmarks during the first year of this activity were:

1) The interoperation between GARUDA and worldwide Grid infrastructure

2) The exploitation of the TEIN3 link for LHC data transfer

3) The exploitation of NKN-TEIN3-GÉANT connectivity for remote control and data collection at the Grenoble beam facility.

With the transition from ERNET to NKN for the network layer an interoperation problem occurred since all the node within the GARUDA grid became not visible to the external world. 
Thanks to the effort, coordinated by the EU-IndiaGrid2 project, of ERNET, NIC and CDAC it was possible to solve this issue in the context of the EU-IndiaGrid2 Workshop in December in Delhi and since end 2010 all the GARUDA infrastructure is visible to worldwide grids. In addition the project supported the interoperability between the European Grid Initiative, EGI [7] and the GARUDA grid infrastructure which is now possible using a metascheduler based on Gridway [8].

The TEIN3 link from Europe to Mumbai was commissioned in March 2010. However a number of issues related to the connectivity between the TEIN2 PoP and the WLCG Tier2 at TIFR needed to be solved. Again with the coordinated effort of NIC and EU-IndiaGrid2 partners it was possible since fall 2010 to exploit the TEIN3 links for LHC data transfers. Considering that the Academia Sinica Computing Centre acts as reference Tier1 for CMS Tier2 at TIFR both TEIN3 links (to Europe for CERN and to Singapore for Academia Sinica Tier1) are crucial for WLCG operation in India. In addition the commissioning of the 1 Gbps NKN connectivity from Kolkata to Mumbai makes the international connectivity available also for the ALICE experiment.

Finally the collaboration between Bhabha Atomic Research Centre (BARC) in Mumbai and Commissariat pour l' Energie Atomique (CEA) in Grenoble represents an excellent showcase for the usage of NKN-TEIN3-GÉANT connectivity for remote control and data collection at the Grenoble beam facility. The BARC and CEA research groups collaborate in experiments dedicated to the study of crystallography of biological macromolecules. using protein crystallography beamlines. Two facilities have been set-up in India allowing to operate remotely the beamline FIP on ESRF, Grenoble. Good data X-ray diffraction data has been collected on crystals of drug resistant HIV-1 protease enzyme. Both BARC and CEA are EU-IndiaGrid2 partners and this activity is fully supported by the EU-IndiaGrid2 project.
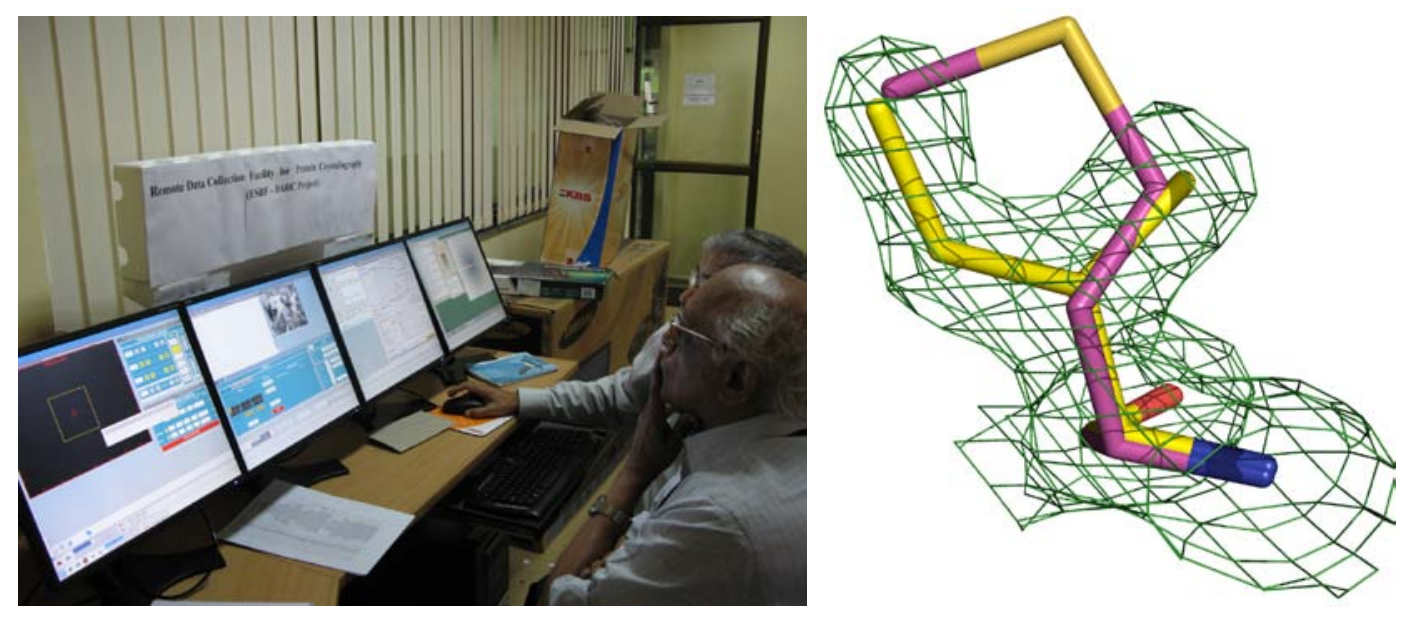

Figure 5 Operation of FIP beamline in Grenoble from BARC Mumbai (left) and example of good quality data collected at FIP and analysed at BARC (Drug-resistant HIV-1 protease enzyme) 


\section{Conclusions}

In conclusion a very positive balance marks the first 15 months of activity of the EUIndiaGrid2 projects thanks to the exceptional development in the domain of e-Infrastructures related to the approval of National Knowledge Network and the launch of TEIN3 connectivity between Europe and India and India to Singapore making Mumbai a vital lynchpin between Europe and Asia. The leading role of EU-IndiaGrid2 partners put the project at the core of these developments and allowed the achievement of significant results for interoperability of European and Indian e-Infrastructures and for the support of Euro-India collaborative research.

\section{References}

[1] The National Knowledge Network, www.nkn.in

[2] GÉANT, the pan-European communications infrastructure www.geant.net

[3] TEIN3, The TransEurasia Information Network http://www.tein3.net/

[4] The WorldWide LHC Computing Grid (WLCG), http://lcg.web.cern.ch/LCG/

[5] See e.g Daniele Buonacorsi, these proceedings

[6] The GARUDA India National Grid Initiative, http://www.garudaindia.in/

[7] EGI, the European Grid Initiative, www.egi.eu and S. Newhouse, these proceedings

[8] The Gridway metacheduler, http://www.gridway.org

\section{Acknowledgments}

I want to express my special thanks to Prof Raghavan, Scientific Secretary to Principal Scientific Adviser to Government of India and Chief Architect and Chairman of Technical Advisory Committee of NKN, for his fundamental contribution to the description of NKN Project. 